\title{
Programas de acceso especial a las carreras de pedagogía: Sus características en las Universidades del Estado de Chile
}

Special access programs of pedagogy careers:

Their characteristics in Chile's State Universities

\author{
Nicolás Hormazabal Gonzáleza ${ }^{a}$, Nicole Abricot Marchant" ${ }^{\mathrm{b}}$, Karen Oyarzo Vargasc, \\ Marlene Alvarado Arteaga ${ }^{\mathrm{d}} \&$ Carolina Bravo Tobar ${ }^{\mathrm{e}}$ \\ a Universidad de Talca. $\varangle$ nihormazabal@utalca.cl. \\ ORCID ID 0000-0001-8344-3517 \\ b Universidad de Santiago de Chile.nicole.abricot@usach.cl. \\ ORCID ID 0000-0003-4539-486x \\ c Universidad de los Lagos. karen.oyarzo@ulagos.cl. \\ ORCID ID 0000-0001-5517-0101 \\ d Universidad de Magallanes. marlene.alvarado@umag.cl. \\ ORCID ID 0000-0001-8108-5613 \\ e Universidad de Talca. carolbravo@utalca.cl. \\ ORCID ID 0000-0003-1062-5824
}

\section{RESUMEN}

Desde el año 2016 la normativa legal chilena establece exigencias específicas para la selección de estudiantes a las carreras de pedagogía, siendo una el ingreso a través de Programas de Preparación y Acceso Especial. La mayoría de las universidades ha definido Programas que cumplen con ese rol, sin embargo, han sido elaborados en diversas perspectivas y con poca claridad respecto de sus consistencias y divergencias. Este artículo pretende caracterizar dichos Programas en las Universidades del Estado. Los resultados del estudio demuestran diversidad de propósitos, modelos de formación y mecanismos de acompañamiento durante la etapa formativa, identificándose dos tendencias generales: capacidades académicas de escritura y matemática, y la identificación de la vocación pedagógica y rol docente en la sociedad. Los hallazgos permiten el progreso de los Programas, considerando la coherencia entre propósitos y diseños metodológicos, además de definir orientaciones para la creación e implementación en otras instituciones de Educación Superior.

PALABRAS CLAVE: Programas de Acceso Especial, Pedagogía, Requisitos de Ingreso a Pedagogía. 


\section{N. Hormazabal et al.}

\section{ABSTRACT}

Since 2016, Chilean regulations establishes specific requirements for students `selection for pedagogical majors, which Special Access and Preparation Programs is one of them. Most of the universities have defined Programs that fulfill this role, however, they have been elaborated in diverse perspectives and with little clarity regarding their consistencies and variations. This article characterizes those Programs from the different Chilean Statal Universities. The results of the study demonstrate diverse purposes, training models and supporting systems during the training stage. It was identified two general trends: academic writing and mathematical skills, and the identification of the pedagogical vocation and teaching role in the society. The findings allow the progress of the Programs considering the coherence between purposes and methodological designs, in addition it provides guidelines for the creation and implementation of this Programs in other Higher Education Institutions.

KEY WORDS: Special Access Programs, Pedagogy, Pedagogy Access Criteria.

\section{INTRODUCCIÓN}

La preocupación por la mejora de la calidad de los aprendizajes de los estudiantes en el contexto escolar ha posicionado a la formación inicial del profesorado como una de las principales cuestiones de quienes pretenden mejorar el sistema educativo en los distintos países del mundo (Darling-Hammond et al. 2009; Ávalos, 2011; Ling \& Mackenzie, 2015).

Durante décadas ha existido consenso en que la calidad de los profesores tiene relación directa con la calidad de la educación, es decir, es uno de los factores más relevantes en el proceso educativo y en el posterior rendimiento de los alumnos (McKenzie \& Santiago, 2005; Hattie, 2009), incluso cuando se controlan variables como aprendizaje previo de los estudiantes y las características de los antecedentes familiares (Darling-Hammond, 2000). En consecuencia, en gran parte de Latinoamérica, a partir de la década de los noventa, se iniciaron procesos de reforma en los sistemas educativos con el propósito de mejorar la calidad de la educación y, al mismo tiempo, dar respuesta pertinente a las diversas exigencias de los cambios sociales, culturales y tecnológicos de la vida moderna (Waissbluth, 2018)

En el caso chileno, conviven una serie de Programas y propuestas educacionales que buscan mejoras educativas, tales como: las leyes N¹9.961 “Evaluación Docente”, N²0.129 que exige "establecer un Sistema Nacional de Aseguramiento de la Calidad de la Educación Superior" y la N²0.903 que crea el "Sistema Nacional de Desarrollo Profesional Docente". También, existen Programas de mejoramiento tales como el Fortalecimiento de la Profesión Docente (desarrollado a fines de 1990), Beca Vocación de Profesor (2011) y la definición de estándares para la Formación Inicial Docente (2011-2012), por mencionar algunos. 
Un estudio sobre percepciones vocacionales de estudiantes de $3^{\circ}$ y $4^{\circ}$ año de Enseñanza Media, provenientes de establecimientos educacionales con buenos resultados académicos, pertenecientes a los tres tipos de administración (municipal, particular subvencionado y particular pagado), posiciona a la carrera de pedagogía y/o educación como la de menor prestigio. Un 66\% de los jóvenes encuestados la sitúa entre las peores valoradas; asimismo, solo un $22 \%$ de los jóvenes está de acuerdo, o muy de acuerdo, que en Chile es un orgullo ser profesor, un 15\% cree que el nivel de los profesores es cada vez mejor y un $5 \%$ afirma que los profesores tienen buenas remuneraciones. El estudio igualmente señala que los propios docentes en ejercicio se consideran desvalorizados, lo que provoca que un alto porcentaje de ellos $(42,1 \%)$ no esté de acuerdo con que sus hijos se dediquen al quehacer docente. Esto indica que, pese al creciente consenso existente respecto al protagonismo e importancia que tienen los docentes en los resultados educativos de los estudiantes, como también en la formación del capital humano, la sociedad aún no les otorga el prestigio que por estas razones merecen, demostrando aún claros síntomas de desvalorización hacia la carrera y el ejercicio profesional. Esto se refleja en la percepción de los estudiantes poseen sobre la profesión docente: un 47\% de las personas manifiesta que no está de acuerdo con que el nivel de los profesores en Chile es cada vez mejor, un $72 \%$ afirma que los profesores de su colegio están bien preparados y tienen interés y dedicación al enseñar $72 \%$ considera que los profesores no tienen buenas remuneraciones (Banco Interamericano del Desarrollo, 2011).

Si bien, entre los años 2000 y 2008, se produjo un aumento del 200\% en la matrícula de estudiantes en carreras de pedagogía, esto se atribuye principalmente al aumento de la oferta de carreras de pedagogía básica y media en Universidades Privadas. Dicho incremento generó un alto nivel de autonomía de la oferta formativa y, a su vez, una desarticulación de ésta con las necesidades del sistema educativo nacional. Condiciones como la baja selectividad de ingreso y las facilidades en el financiamiento, como por ejemplo el Crédito con Aval del Estado (CAE), transformaban a las carreras de pedagogía en una oportunidad para estudiantes provenientes de sectores sociales que accedían por primera vez a la Educación Superior (García-Huidobro, 2011; cf. Rodríguez \& Castillo, 2014).

Dado lo anterior, surgen dificultades tanto en la regulación de la oferta formativa docente, como en los mecanismos de selección de los estudiantes que ingresan a las carreras de pedagogía. Si bien, se implementan diversas estrategias, tales como: destinar recursos a cambio de compromisos de mejora en Programas de Fortalecimiento de la Formación Inicial Docente (FFID) entre los años 1997 y 2002; posteriormente los Programas de Mejoramiento de la calidad y equidad en la educación terciaria (MECESUP), en los que se realizaron cambios a los currículos de formación según lo requerido, nunca hubo un cierre adecuado de los proyectos ni una evaluación de su efecto en el tiempo (Ávalos, 2014).

Lo anterior evidencia que la desvalorización de la carrera profesional docente, en parte, se debe a la ausencia de políticas educativas claramente direccionadas, consistentes 


\section{N. Hormazabal et al.}

y permanentes, que regulen tanto el acceso de los estudiantes como la pertinencia de las instituciones de Educación Superior que imparten las carreras de pedagogía.

Tal como se menciona en el Informe McKinsey (Barber \& Mourshed, 2008), la valoración social y prestigio de la profesión docente afecta directamente la calidad de los estudiantes que la carrera es capaz de captar, así como la calidad de los profesores que egresan. En esta línea, durante el año 2016 entra en vigencia la Ley N²0.903, la que crea el Sistema Nacional de Desarrollo Profesional Docente, cuyo objetivo es reconocer la docencia, apoyar su ejercicio laboral y también aumentar la valoración social de la profesión. Esta ley establece mecanismos específicos para captar a estudiantes con destacadas trayectorias académicas que manifiesten interés y vocación temprana con la carrera profesional docente; como también para retener a los mejores profesores, incentivando su desarrollo profesional bajo el "Sistema de Carrera Profesional Docente".

De esta forma, el Ministerio de Educación (en adelante MINEDUC) propone que las universidades diseñen e implementen mecanismos especiales de difusión, admisión y matrícula para las carreras de pedagogía, con la finalidad de promover la equidad e inclusión en el acceso a la Educación Superior. Esta estrategia se sustenta en la educación como derecho social, en la idea lógica que los talentos se encuentran distribuidos de igual forma en toda la población y que particularmente la vocación pedagógica no es exclusiva de un sector en específico, pudiendo ser desarrollada en cualquier tipo de dependencia y contexto educativo.

En concordancia con lo anterior, esta ley señala que: a) podrán ingresar a carreras y Programas de pedagogía los estudiantes que hayan realizado y aprobado un programa de preparación y acceso de estudiantes de educación media para continuar estudios en la Educación Superior, reconocido por el MINEDUC y habiendo rendido la Prueba de Selección Universitaria (en adelante PSU), y b) podrán ingresar a estos Programas todos los estudiantes de enseñanza media que demuestren condiciones académicas y/o talento temprano para ejercer la profesión docente. Bajo este marco regulatorio, las vías de acceso y sus exigencias irán experimentando cambios progresivos hasta el año 2023, en donde, por ejemplo, junto con aprobar el programa de acceso, los estudiantes que ingresen por esa vía también deberán encontrarse, paralelamente, dentro del 15\% con mejor rendimiento de su generación, al momento de egresar de Enseñanza Media (Ley N²0.903).

En este nuevo contexto, las universidades han desarrollado e implementado este tipo de Programas de Acceso Especial a las carreras de pedagogía, después de haber recibido el reconocimiento oficial del MINEDUC. Los Programas han sido diversos en propósitos, métodos y otros elementos; sin embargo, durante su implementación, no se ha sistematizado la experiencia lograda, ni las fortalezas y debilidades identificadas, como para reconocer las diversas formas en que las universidades han abordado esta política pública.

Como respuesta a este requerimiento de levantamiento de información y conocimiento, este artículo describe y analiza comparativamente el diseño de los Programas de Acceso 
Especial de las Universidades del Estado de Chile ${ }^{1}$, para comprender cómo estas instituciones han respondido al desafío impuesto por esta política pública. Además, se identifican algunas controversias en sus diseños, como también ejemplos de buenas prácticas formativas logradas en el marco de su implementación.

\section{MARCO TEÓRICO}

La formación docente ha sido foco de atención en la opinión pública, en las políticas educativas y en la investigación global y local. Parte de los análisis esgrimidos para el caso chileno sobre los Programas de formación docente declaran que no están atrayendo "a buenos egresados de la educación media, y que los Programas de formación han crecido más en número que en la calidad de las instituciones formadoras, lo que afectaría la competencia de sus egresados" (Ávalos, 2014, p. 12).

En este contexto, una de las grandes interrogantes de la política pública orientada a la formación de profesores ha sido: ¿Cómo captar a los mejores estudiantes para que ingresen a las carreras de pedagogía? Esta pregunta ha tenido diversas respuestas a nivel internacional.

La necesidad de mejorar la formación de profesores ha derivado en la idea de que es necesario atraer gente talentosa a la docencia (Barber \& Mourshed, 2008). Sin embargo, estas pretensiones se encuentran con un escenario de baja valoración social de la enseñanza, la cual, a pesar de múltiples esfuerzos continúa siendo un desafío de los sistemas educativos en distintas partes del mundo (Ticky, 2016). A nivel internacional, la falta de fuerza laboral docente ya sea en general o en áreas específicas (Borgerding, 2015), ha inspirado al diseño de políticas públicas a fin de captar el interés hacia las pedagogías, y recomendaciones de organismos internacionales para disminuir la escasez de profesores (UNESCO, 2013). En este escenario, los procesos de atracción y selección de los mejores postulantes para las carreras de pedagogía son diversos y ajustados a los diferentes contextos, según reporta la experiencia internacional.

Por ejemplo, en Dinamarca, reclutar un número suficiente de docentes capaces se ha convertido en una preocupación creciente en los últimos años, durante los cuales el número de solicitantes ha disminuido constantemente. En el caso de Finlandia, sigue teniendo un gran número de solicitantes y una entrada muy selectiva en el que solo alrededor del $14 \%$ de los postulantes es aceptado (Reimer \& Dorf, 2014). Por otro lado, en el mismo país, la formación docente es un programa de maestría de cinco años que solo se puede estudiar en la universidad, mientras que en Dinamarca los profesores reciben capacitación en centros de formación docente por un período de cuatro años y obtienen un título de licenciatura profesional (Reimer \& Dorf, 2014).

Se reconoce la existencia y desarrollo de programas diseñados por Universidades Privadas, los que no se consideran para este estudio, debido a que se realiza en el marco del CUECH. 


\section{N. Hormazabal et al.}

Respecto a los requisitos de ingreso, las experiencias analizadas se refieren a las competencias académicas y a las experiencias tempranas en el campo. Así, en Australia, se declara que los postulantes a la formación docente provengan del 30\% mejor en las áreas de lenguaje y matemática (Gore et al. 2016). Por otro lado, en Portugal y en Suecia, alineados con el Plan Bolonga, se requiere un grado de magíster para ser profesor, y se enfatiza una primacía del conocimiento disciplinar junto a un enfoque basado en la investigación para la enseñanza y la práctica docente (Flores \& Niklasson, 2014).

La realidad de Singapur, sin embargo, es distinta. Se sugieren experiencias tempranas en el campo por considerar que reflejan mejor las capacidades de los postulantes a pedagogía y permiten evaluar las actitudes y aptitudes de los candidatos y asegurarse de que el sistema reclute a las personas más apropiadas, dando la oportunidad de afirmar su propio interés e idoneidad para la enseñanza; proporcionar experiencias escolares de la vida real, que los preparan no solo para la capacitación como docentes sino también para desafiar las realidades escolares ( $\mathrm{Ng}$ et al. 2018).

\section{Antecedentes}

En Chile, a diferencia de países como Singapur, durante las últimas décadas, la selección a las carreras de pedagogía se asocia al egreso de la enseñanza secundaria a través de pruebas de selección universitaria (iguales para todas las carreras del sistema universitario) o a un ingreso directo, no selectivo, a algunas instituciones de Educación Superior (Ávalos, 2014).

Antes del año 2016, estas universidades no selectivas habían alcanzado un porcentaje de la matrícula de estudiantes de pedagogía muy superior a las universidades selectivas, demostrando niveles de calidad general muy por debajo de los presentados en las instituciones selectivas (Ávalos, 2014). Sin embargo, después de la implementación de la Nueva Ley de Carrera Docente (Ley N²0.903) las carreras de pedagogía y su matrícula han descendido, particularmente en las instituciones que no cumplían con criterios de calidad mínimos definidos en el sistema (Arias \& Villarroel, 2019). A pesar del positivo efecto que se esperaba lograr en la formación de profesores, las proyecciones señalan un escenario complejo, indicando que en el año 2023 el número de profesores que se titulará en Chile caerá en un 45\% (AEqualis, 2019).

A estos antecedentes de disminución de matrícula de las carreras y del egreso, la autora Beatrice Ávalos indica que si bien el problema de la escasez de profesores afecta principalmente a ciertas áreas (CIAE, 2018), la dificultad mayor es la baja retención en el sistema escolar. La evidencia indica que alrededor de un 40\% de los docentes abandona la profesión durante los primeros años de ejercicio de la carrera (Valenzuela \& Sevilla, 2013, realizado en el marco del proyecto Fondecyt $\mathrm{N}^{\circ} 1120740$ ).

Ese antecedente desafía al sistema para poner especial atención a la oportunidad que 
los Programas de Acceso otorgan para captar estudiantes con alto potencial y convertirlos en profesores de excelencia, que permanezcan comprometidos con su formación y la de sus estudiantes.

\section{El citado concepto de la vocación pedagógica}

Sin duda, una de las ideas subjetivas más recurrentes y controvertidas respecto de qué buscar para captar a los buenos profesores, es la recurrente categoría de "vocación pedagógica”. Según Araya y Wong (2013), los Programas de Acceso Especial a las pedagogías indican que la "vocación" es la motivación intrínseca que lleva a los estudiantes a ingresar a estudiar educación. La "vocación", siguiendo a los autores, se traduce en la identidad y compromiso del futuro educador con la enseñanza. Estos Programas consideran a la "vocación pedagógica" como un factor potenciador del rendimiento y de la identificación del estudiante de pedagogía con su carrera (Araya \& Wong, 2013, p. 3).

La importancia de analizar en forma sistemática el concepto de "vocación pedagógica" parece evidente en el marco de una iniciativa de política pública orientada a la cualificación de la formación integral de los futuros profesores. El concepto da cuenta de procesos que se verifican en el terreno de la subjetividad docente y que movilizan poderosamente tras la opción por el estudio y el ejercicio de la pedagogía. Dada su relevancia, la "vocación pedagógica" ha estado presente en la historia de la educación en Chile, formando parte de los discursos de la política pública y de las diversas comprensiones sobre el "ser docente" que circulan en la sociedad (Núñez, 2007).

Sumado a lo anterior, el autor aborda el desarrollo de la identidad del profesorado señalando que corresponde a un proceso dinámico que se cristaliza en distintas identidades a través de la historia, manteniendo una fuerte relación con las ideas dominantes en la sociedad acerca de lo que es "ser docente". Para el autor, si bien en cada época han surgido nuevas comprensiones, éstas han convivido con rasgos que definieron la identidad colectiva del profesorado en tiempos anteriores.

En cuanto al desarrollo teórico del concepto "vocación", se puede distinguir una tradición evolutiva, la cual "supone que existe dentro del desarrollo del sujeto un proceso de elección vocacional ligado intrínsecamente a la constitución de su propia identidad y que se manifiesta en conductas concretas orientadas a su consecución" (Quintana, 2014, pp. 1314). Desde esta mirada, el proceso de la elección vocacional se visualiza como algo continuo, que deriva de una serie de decisiones y experiencias de vida. Es por ello, que se distinguen elecciones muy tempranas, en donde el individuo no ha alcanzado aún su maduración y que tienden a estar muy cargadas de fantasía de aquellas que tienen relación con un proyecto de ser y quehacer (García-López, 2003).

Los aportes del campo de la psicología a esta perspectiva evolutiva, principalmente en el estudio de los componentes de la vocación por medio de la psicometría, abren un enfoque del 


\section{N. Hormazabal et al.}

estudio de "rasgos y factores". El conocido Test de Holland (1977) podría situarse a partir de esta tradición. Para este autor, la elección vocacional refiere a una manifestación conductual que expresa conocimientos adquiridos, motivaciones, características de la personalidad y aptitudes.

Desde un enfoque interactivo - psicosocial, el concepto habitualmente denominado "vocación" se comprende más bien como la construcción de rasgos de identidades profesionales, derivadas de experiencias de los sujetos en su relación con el medio social (García-López, 2003). En cierta sintonía con este enfoque, Foladori (2009) sugiere un análisis dialéctico de la sociedad y psicoanalítico del sujeto para abordar lo vocacional, específicamente, desde aspectos individuales, su interrelación con contextos familiares y nacionales, así como también con los ámbitos globales en donde la elección vocacional no se acaba en reconocer intereses o habilidades personales, sino que se enlaza con el contexto sociocultural y económico de un determinado tiempo histórico.

\section{DISCUSIÓN BIBLIOGRÁFICA}

En relación a los factores que inciden en la elección de la pedagogía como carrera universitaria, la literatura ha explorado aspectos relacionados como las motivaciones, habilidades académicas, no académicas y orígenes socioculturales de los estudiantes que deciden ser profesores.

Una línea de investigación específica se ha conformado a partir de las motivaciones de los estudiantes para convertirse en profesores. En ese contexto, la investigación señala motivaciones intrínsecas, altruistas y extrínsecas, siendo las de tipo intrínsecas y altruistas mayormente nombradas por ellos al momento de identificar los principales intereses para escoger la pedagogía en distintos países, contextos y áreas de enseñanza. Si bien aquellas de tipo extrínseco están menos presentes, ejercen un rol en la atracción a la docencia. Por ejemplo, Neugebauer (2015) estudió las tendencias en el mercado laboral docente y las características de las cohortes de tres décadas en Alemania, concluyendo que es la motivación intrínseca la que atrae a los estudiantes a la docencia, incluso cuando las condiciones laborales son difíciles. El estudio de Ng, Lim, Low \& Hui (2018) releva la "pasión por la enseñanza”, entendida como una motivación intrínseca en su investigación ya que, y de acuerdo con sus antecedentes, la pasión y el compromiso con la pedagogía se vinculan con mayor resiliencia y permanencia en la profesión. No obstante, aclara que esta pasión debe comprenderse en distancia de un interés superficial por enseñar, por lo cual debe ser "puesta a prueba" a través de un ejercicio real, ya que un interés superficial no contribuye finalmente a sostener la motivación al momento de enfrentar situaciones desafiantes.

Estos planteamientos se contradicen con los resultados de Manuel \& Carter (2016), los cuales concuerdan en identificar las motivaciones altruistas e intrínsecas como factores influyentes en la decisión de ser docente. Sin embargo, el rol que le conceden a los contextos 
de ejercicio interviene en la mantención de la motivación inicial, es decir, para una proporción significativa de su muestra, sus aspiraciones, expectativas y metas iniciales que habían sido mermadas por fuerzas del contexto tales como las presiones asociadas a preparar a los estudiantes para exámenes nacionales o la marginalización de la toma de decisiones vinculadas a su práctica de aula.

Continuando con las motivaciones altruistas, una de las razones más frecuentes para que surja el interés en la enseñanza es trabajar con niños o adolescentes (Flores \& Niklasson, 2014; Giersch, 2016; Gore et al. 2016). En el caso de estudios desarrollados en Portugal y Suecia, este interés altruista puntuaba como el factor más decisivo de su muestra, seguido por la adquisición de conocimientos en un campo determinado para Portugal, y contribuir a la sociedad, en el caso de Suecia. Sumado a lo anterior, estudios desarrollados con estudiantes finlandeses y daneses enfatizaron en los aspectos intrínsecos de la docencia, donde el contenido educativo y social de la enseñanza fue más valorado por los primeros, mientras que los segundos valoraban más la compatibilidad horaria y familiar de la docencia (Reimer \& Dorf, 2014).

En relación a las motivaciones extrínsecas, la seguridad laboral y las oportunidades de empleo son las más destacadas (Flores \& Niklasson, 2014; Giersch, 2016), así como también la compatibilidad horaria y familiar (Reimer \& Dorf, 2014; Giersch, 2016), aunque éstas en ningún estudio son enunciadas dentro de la razón preponderante para estudiar pedagogía.

Según Vaillant (2013), "en América Latina, la docencia se ha transformado en una profesión que no atrae a los mejores candidatos. Quienes ingresan a las Universidades y a los Institutos de Formación tienen en promedio, peor historial educativo que otros estudiantes que acceden a otros estudios más valorizados socialmente" (p. 199).

Respecto del origen social de los futuros profesores, Palazzo y Gomes (2012), a través de un estudio de caso en Brasil, compararon la situación social, económica y cultural de Programas de formación de profesores con Programas de formación en leyes. Su objetivo era verificar la existencia de diferencias sociales entre los alumnos, de estos cursos, dentro de la institución, a pesar de la expansión y democratización de la educación superior en Brasil. Los autores concluyen que los futuros educadores brasileños provienen de estratos socioeconómicos menos privilegiados y, por tanto, tienen menos capital cultural a diferencia del curso de leyes.

Según Bruns y Luque (2014), en Latinoamérica los profesores son también más pobres que el conjunto general de estudiantes universitarios. Los datos sobre ingreso a las universidades muestran que los estudiantes que cursan carreras relacionadas con la educación son de condición socioeconómica más baja y tienen más probabilidades de pertenecer a la primera generación de sus familias con estudios universitarios, que quienes ingresan en otras carreras. Los datos señalan un conjunto de estudiantes que posiblemente ha tenido escaso contacto con otras profesiones a lo largo de su vida y, en consecuencia, tiene aspiraciones académicas más limitadas. 


\section{N. Hormazabal et al.}

En este mismo escenario, quienes ingresan a las carreras de pedagogía son académicamente menos preparados que el conjunto general de los estudiantes de la Educación Superior (Bruns y Luque, 2014). Los alumnos de 15 años que manifiestan estar interesados en la docencia tienen puntajes comparativamente más bajos en las pruebas PISA (Programa para la Evaluación Internacional de Estudiantes) de matemática que los que muestran interés por la ingeniería, en todos los países de la región. Las evidencias disponibles muestran que América Latina no atrae a los mejores candidatos que necesita para conformar sistemas educativos de primer nivel.

Respecto del capital cultural de los estudiantes de pedagogía en nuestro país, Mizala et al. (2011) caracterizaron a los estudiantes de pedagogía chilenos a partir de un estudio de carácter mixto. Los resultados muestran que el estudiante tipo multivariable es una mujer, de bajo rendimiento académico, de origen socioeconómico vulnerable y que realizó sus estudios básicos y secundarios en un establecimiento municipal, principalmente de rama técnico profesional de menor calidad académica, proveniente de hogares donde los padres tienen niveles educacionales e ingresos familiares inferiores a los padres de los matriculados en otras áreas.

Los estudiantes que se matriculan en una carrera universitaria en educación tienen puntajes SIMCE, PSU, notas de enseñanza media (NEM) y Ranking de notas significativamente inferiores en términos estadísticos a los que se matriculan en otras áreas (Mizala et al. 2011). En otro estudio, Cabezas y Claro (2011) caracterizan al estudiante de pedagogía en Chile como postulante con un puntaje promedio 30\% inferior al de medicina en la Prueba de Selección Universitaria (PSU).

Ruffinelli y Guerrero (2009) estudiaron el destino laboral de egresados de pedagogía en Educación Básica. Los resultados de su estudio les permitieron comprobar que la segmentación e inequidad del sistema educativo chileno configura un escenario en el que se estarían formando docentes explícitamente de acuerdo a su origen socioeconómico para volver luego a impartir clases al mismo sistema educativo en el que fueron formados en la enseñanza regular. Para estos autores, esta segmentación y segregación educativa niega la posibilidad de cohesión social necesaria para corregir inequidades y lograr aprendizajes en un contexto integrador donde compañeros de aula y docentes cuenten con un capital social y cultural distinto del propio. De este modo, según los autores, los postulantes con mayor capital cultural transmitido por el hogar, que además cuentan con mayor capital escolar acumulado en su trayectoria académica, son los que acceden a las universidades de mayor reputación, lo que facilita su acceso al mercado laboral y una movilidad hacia ocupaciones de mayor reputación y remuneración, estratificándose así también el mercado ocupacional de destino.

Las problemáticas anteriormente mencionadas, como la deserción docente, vendrían de algún modo a intentar resolverse a partir de la promulgación de la Ley №20.903 que crea el Sistema Nacional de Desarrollo Profesional Docente en el año 2016. Esta política define la posibilidad de que las universidades diseñen e implementen Programas de Acceso Especial a las carreras de pedagogía, a fin de captar, seleccionar y formar a los estudiantes de Educación 
Media interesados en la profesión docente, en el marco de Programas que complementan su educación secundaria.

En este contexto, el artículo analiza las principales características de los Programas de Acceso Especial implementados en la actualidad en las Universidades del Estado de Chile. Adicionalmente, identifica algunas controversias en el diseño de los Programas, complementadas con algunas experiencias importantes para tener en cuenta en su evaluación.

\section{MÉTODO}

A través de un proceso de investigación mixto de alcance exploratorio, se realizó un análisis documental, trabajando con los formularios de postulación de 11 instituciones pertenecientes a la Red de Universidades Estatales de Formación Inicial Docente y una encuesta para comparar los Programas de Acceso Especial a las Carreras de Pedagogías de las Universidades del Estado, que habían sido reconocidos ante el Ministerio de Educación de Chile desde el año 2017.

En una primera fase, se solicitaron los informes de reconocimiento de cada una de las instituciones, lo que permitió contar con 11 Universidades que hasta la fecha de inicio del estudio contaban con Programas reconocidos por el MINEDUC. Las resoluciones exentas contenían información detallada y se realizó análisis de criterios comunes en las dimensiones del diseño, implementación y evaluación global de los Programas, obteniendo variables cuantitativas sobre el tipo de Programa, sus objetivos, módulos, equipos, mecanismos de selección, procesos de acompañamiento, entre otros elementos, realizándose para ello una agrupación de criterios comunes en relación a los 11 informes de reconocimiento de los Programas. En una segunda fase, y a modo general, se empleó un formulario complementario de 22 preguntas abiertas tipo encuesta, cuyo objetivo fue recabar información adicional para ampliar la descripción del diseño, implementación y evaluación de los Programas; dicho formulario fue respondido por cada uno de los representantes de las Universidades en la mesa de Programas de Acceso en el trabajo en Red, obteniendo respuesta de 6 de ellas. Por ende, la sistematización de información adicional se utilizó para complementar las características generales de los Programas.

El análisis se realizó a través de una sistematización comparativa que identificó patrones comunes y diferenciadores en el diseño e implementación de cada uno de los Programas. Dicha información fue procesada y reportada en términos de sus frecuencias o contenidos, considerando categorías predefinidas.

Sobre la base de lo anterior, se realizó un análisis de los elementos y la comparación de los programas, sus convergencias y divergencias sobre los propósitos, diseños, implementación y procesos de acompañamiento que se realizan con los estudiantes en las carreras de pedagogía. La presentación de los resultados se estructura a partir de los distintos elementos que componen los Programas de formación. 


\section{N. Hormazabal et al.}

\section{RESULTADOS}

\section{Objetivos de los Programas}

Los Programas de formación declaran diversos tipos de objetivos y entre ellos es posible identificar dos grandes perspectivas. Algunos se focalizan en la nivelación académica de los estudiantes que están interesados en estudiar pedagogía y que han demostrado mérito y persistencia en contextos de alta vulnerabilidad social; por ejemplo, el objetivo de uno de los Programas es "promover el acceso y acompañamiento académico y socioafectivo de estudiantes preferentemente vulnerables, con talento y buen desempeño escolar en contexto, a carreras pedagógicas". Otros, no se direccionan hacia estudiantes vulnerables, sino que tienen una orientación esencialmente vocacional e intentan captar aquellos interesados en estudiar pedagogía para fortalecer su motivación en esta área profesional. Uno de los Programas que posee esta orientación declara el siguiente objetivo: "Fortalecer la valoración de la docencia y el desarrollo académico de estudiantes de educación media que demuestren alto rendimiento académico en contexto y vocación pedagógica temprana, con el fin de favorecer la exploración vocacional y la formación de futuros profesores con proyectos de vida vinculados a la transformación social, la innovación y la formación continua”.

$\mathrm{Al}$ analizar la totalidad de los Programas, es posible identificar una u otra perspectiva en sus propósitos; sin embargo, la mayoría de ellos - incluso aquellos de orientación a la nivelación académica - enfatiza aspectos actitudinales relacionados con la vocación, talento, motivación o interés por la enseñanza.

Enadelante se sintetizanlos principales objetivos contenidos enlos Programas: 1) promover la equidad, diversidad y calidad de la enseñanza en la Educación Superior, específicamente en las carreras y Programas de pedagogía, así como generar nuevas perspectivas y oportunidades en los estudiantes de enseñanza media; 2) captar tempranamente estudiantes con interés y condiciones para ejercer las funciones de los profesionales de la educación en establecimientos educacionales; 3) generar colaboración entre los establecimientos escolares y las instituciones de Educación Superior; y por último, 4) preparar tempranamente a los estudiantes para la vida universitaria, acompañarlos en su inserción a la Educación Superior y otorgarles estrategias de nivelación académica y seguimiento a fin de garantizar su permanencia exitosa en la universidad, lograr su titulación y su posterior ejercicio profesional docente.

\section{Admisión a los Programas}

Sobre la admisión de estudiantes del sistema escolar a los Programas de Acceso Especiales, se reconocen diversos mecanismos, los que van desde la solicitud de antecedentes sobre variables académicas de los estudiantes, hasta la búsqueda de evidencias sobre su interés específico por estudiar pedagogía. A continuación en el gráfico (Fig. 1), se presentan 
Fig. 1. Mecanismos de admisión de los Programas.

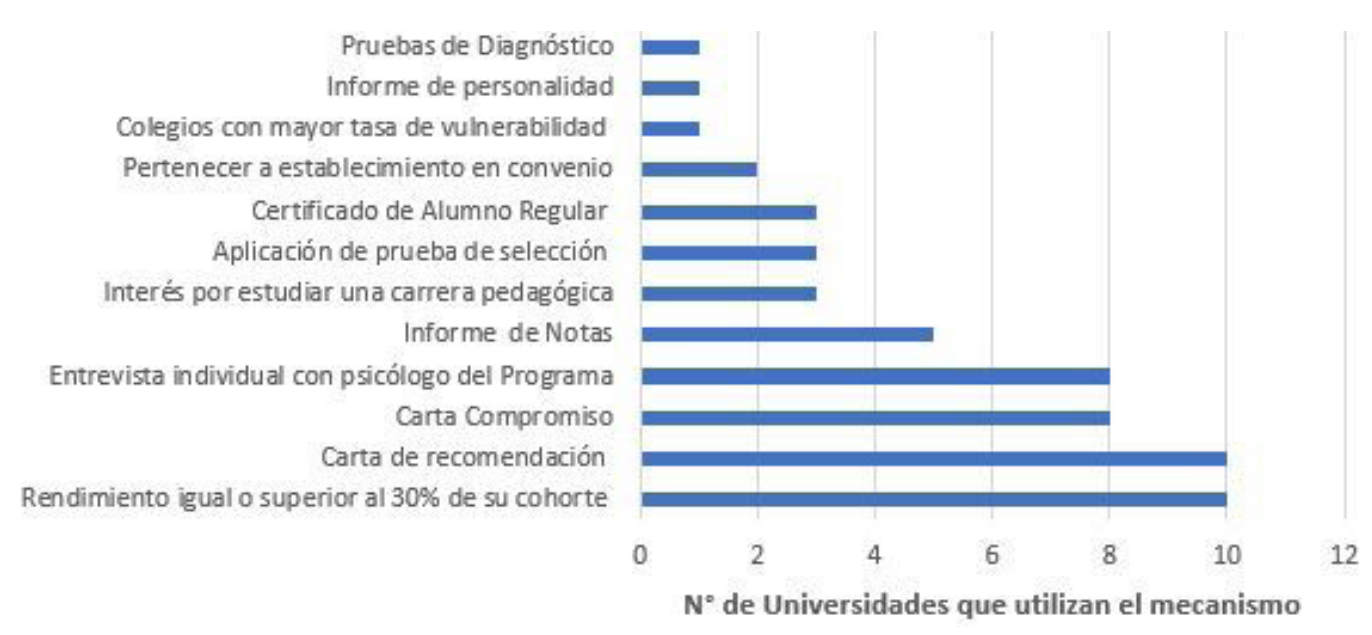

Fuente: Elaboración propia.

los mecanismos de admisión a los Programas (eje Y) que utilizan las 11 Universidades (eje X) que entregan información a través de los formularios de investigación.

En el gráfico se aprecia que la mayoría de las universidades, 10 de las 11, considera en su proceso de postulación, el rendimiento escolar de los estudiantes en contexto de su cohorte y una carta de recomendación por parte de la entidad educativa. A su vez, emplean, en menor número, los mecanismos de ingreso como pruebas de diagnóstico, informes de personalidad y proveniencia de establecimientos con mayor tasa de vulnerabilidad. Solo una de estas instituciones utiliza estos últimos mecanismos.

Se destaca que todas las universidades realizan esfuerzos específicos para la difusión de los Programas. Sin embargo, algunas de ellas efectúan llamados masivos en medios de comunicación a estudiantes interesados en participar, mientras que otras focalizan su atención en centros escolares vinculados a las casas de estudios a través de convenios de colaboración de diversa naturaleza.

En las universidades que focalizan su atención en centros escolares específicos, es posible identificar procedimientos muy definidos. Por ejemplo, realizan una exploración sistemática de los establecimientos educativos de la Región, provincia o ciudad, incluyendo un catastro que permite, posteriormente, construir un perfil de cada establecimiento en función de la cantidad de estudiantes, tipo de financiamiento, sello, promedio PSU, etc. Estos datos se complementan con un análisis de distancia geográfica, a la redonda, de los establecimientos en relación con la Universidad o campus. Desde la selección de éstos, se programan acciones clave. Realizan visitas iniciales que permiten el contacto de integrantes del equipo del Programa con directores, orientadores o profesores jefes, quienes serán agentes articuladores para la detección de estudiantes con motivación, interés y vocación por la pedagogía. En la misma línea, se realizan reuniones con Padres y Apoderados con el propósito de presentar objetivos, requisitos de aprobación, mecanismos de acompañamiento 
estudiantil en el Programa y las acciones contempladas para apoyar su posterior ingreso a las carreras de pedagogía. Después de estos procesos de difusión, los estudiantes están en condiciones de postular a los Programas.

\section{Mecanismos de Diagnóstico}

Con el fin de detectar las brechas entre el perfil esperado por las instituciones para ingresar al Programa de Acceso Especial y las condiciones reales de acceso de los estudiantes, las 11 instituciones realizan actividades específicas para diagnosticar sus condiciones de entrada. Como se plantea en la Fig. 2, el proceso de diagnóstico se estructura y organiza en ámbitos disciplinares, características personales y variables vocacionales.

Fig. 2. Mecanismos de diagnóstico de los Programas.

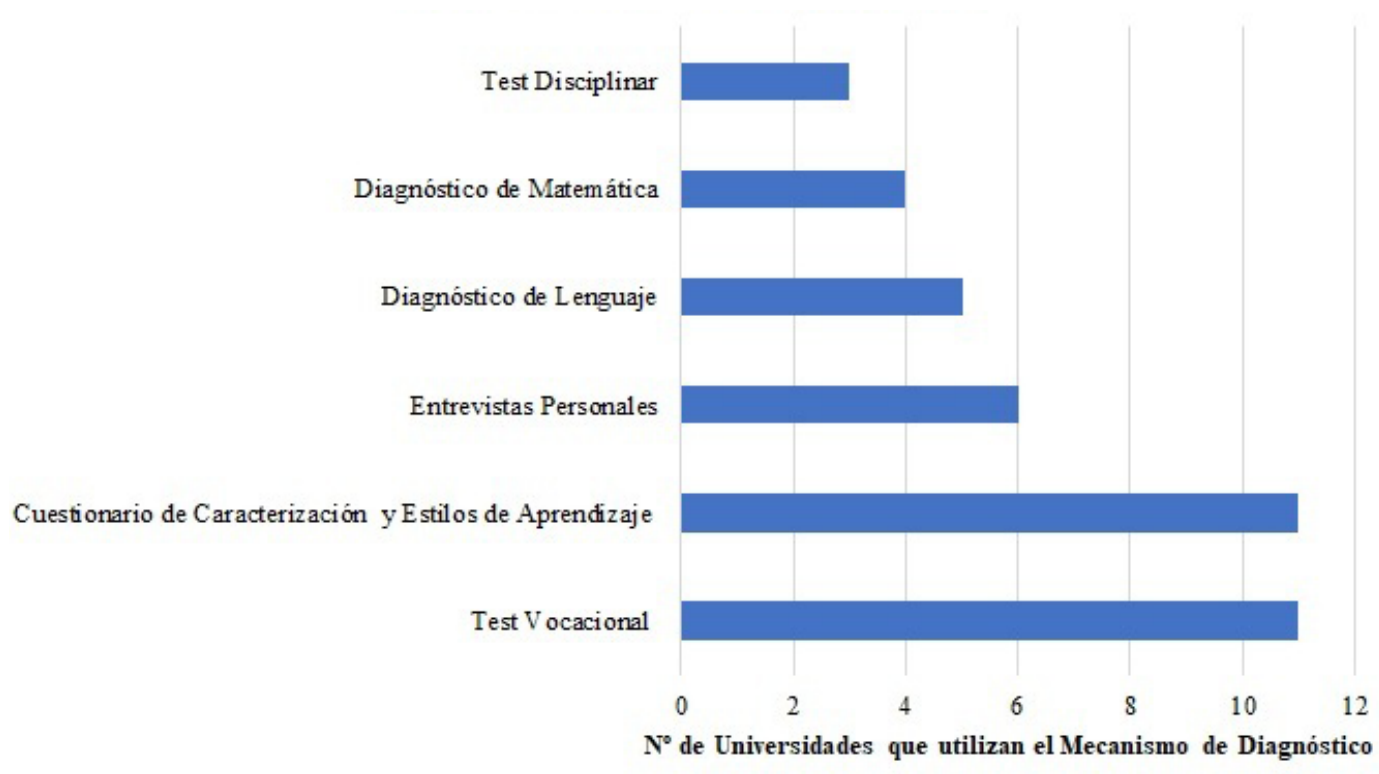

Fuente: Elaboración propia.

La gráfica muestra que el 100\%de las instituciones utiliza Cuestionarios de Caracterización, instrumentos de reconocimiento de Estilos de Aprendizaje y Test Vocacionales. Por otra parte, el 56\% de ellas aplica Entrevistas Personales, un 45\% evalúa manejo de Lenguaje, un 36\% valora habilidades Matemáticas y un 27\% aplica Test de Carácter Disciplinar.

\section{Actividades y Módulos de preparación}

En el gráfico de la Fig. 3, respecto a las actividades anuales que contemplan los Programas, éstas corresponden a módulos, cursos y/o talleres que se imparten en las 11 Universidades. La sumatoria de éstas corresponde a un total de 95 actividades, las cuales son de carácter académico, formativo/vocacional y recreativo/cultural. 
Fig. 3. Tipos de actividades de los Programas.

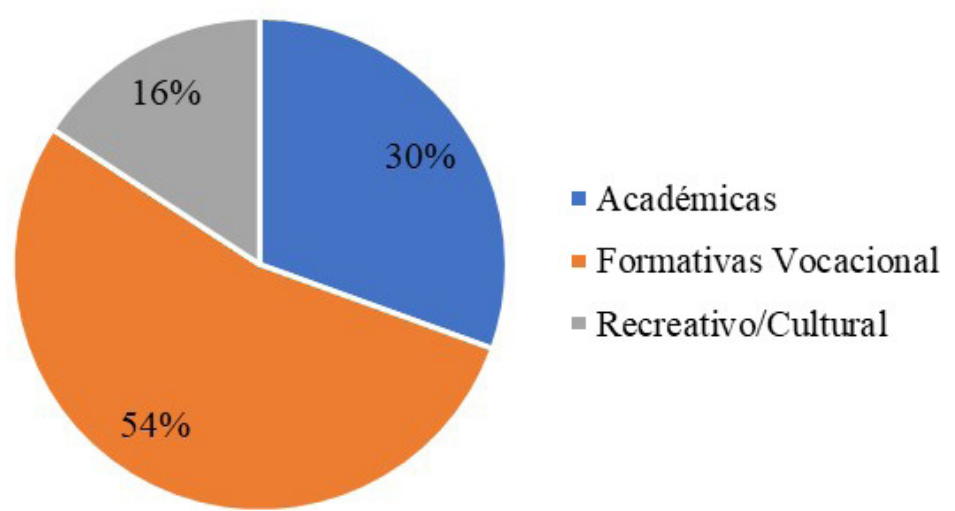

Fuente: Elaboración propia.

De las actividades de los Programas en la Fig. 3, el 30\% de ellas se concentra en el área Académica correspondiente a 29 actividades, el 54\% en el ámbito Formativo Vocacional correspondiente a 51 actividades y el 16\% en el Recreativo/Cultural correspondiente a 15 actividades.

Lo anterior muestra una diversidad de actividades que compone los Programas, concentrándose mayoritariamente en aquellas de tipo Formativo Vocacional.

A continuación (Fig. 4), se especifican las actividades contempladas en los diferentes ámbitos.

Fig. 4. Detalle de actividades de los Programas.

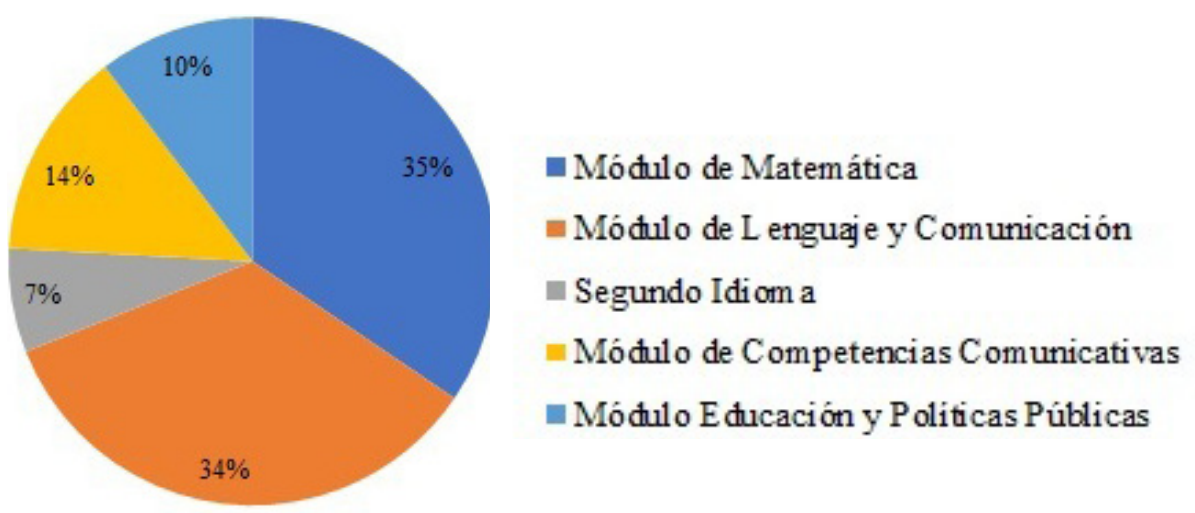

Fuente: Elaboración propia.

En el gráfico de la Fig. 4, en el ámbito Académico, de las 29 actividades de las 11 Universidades, alrededor del $69 \%$ de aquéllas se asocia a Matemática y Lenguaje y Comunicación, lo que marca tendencia. Un 14\% refiere a Competencias Comunicacionales, un $10 \%$ a temas referidos a Educación y Políticas Públicas, mientras que un $7 \%$ se orienta a aprendizaje de Segundo Idioma. 


\section{N. Hormazabal et al.}

Fig. 5. Detalle de actividades Formativo-Vocacionales de los Programas.
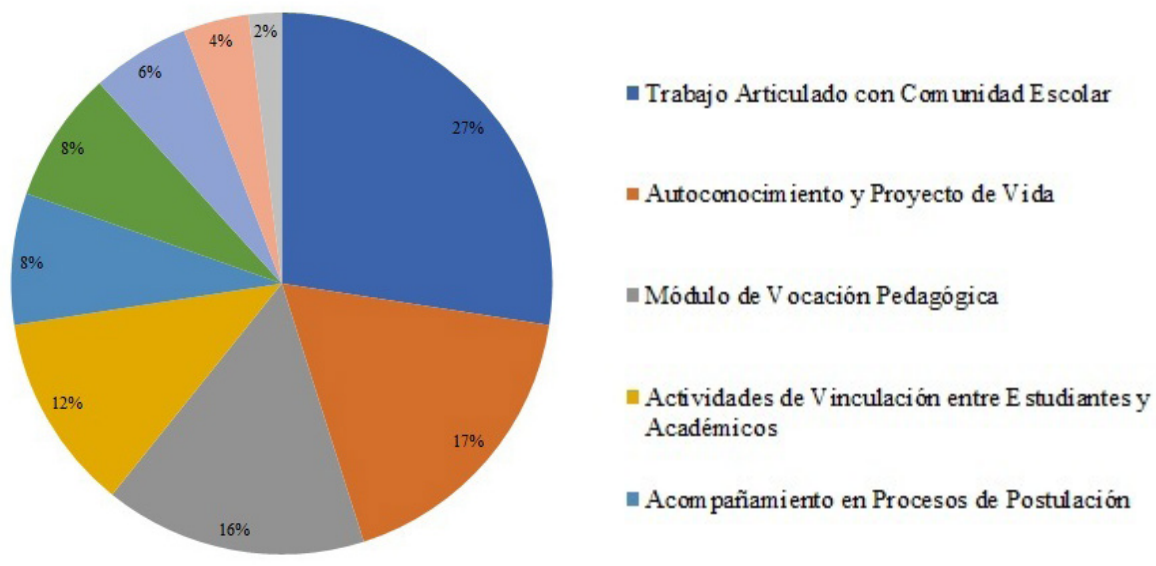

Fuente: Elaboración propia.

En el gráfico de la Fig. 5, en el ámbito Formativo-Vocacional de las 51 actividades en las 11 Universidades, el 27\% de éstas refiere a Trabajo Articulado con la Comunidad Escolar, un $17 \%$ se orienta a Autoconocimiento y Proyecto de Vida y un 16\% a Vocación Pedagógica. A su vez, un 12\% corresponde a acciones de Vinculación entre Estudiantes y Académicos, un 8\% a Gestión Personal y otro 8\% a acciones de Acompañamiento para el Proceso Postulación e ingreso a la Universidad, un 6\% refiere a Fortalecimiento Vocacional, un 4\% a Desarrollo de Habilidades Sociales y un 2\% a actividades de Enriquecimiento Psicosocial. El foco de este ámbito está en el vínculo con la escuela y Autoconocimiento.

Fig. 6. Detalle de actividades Recreativo-Culturales de los Programas.

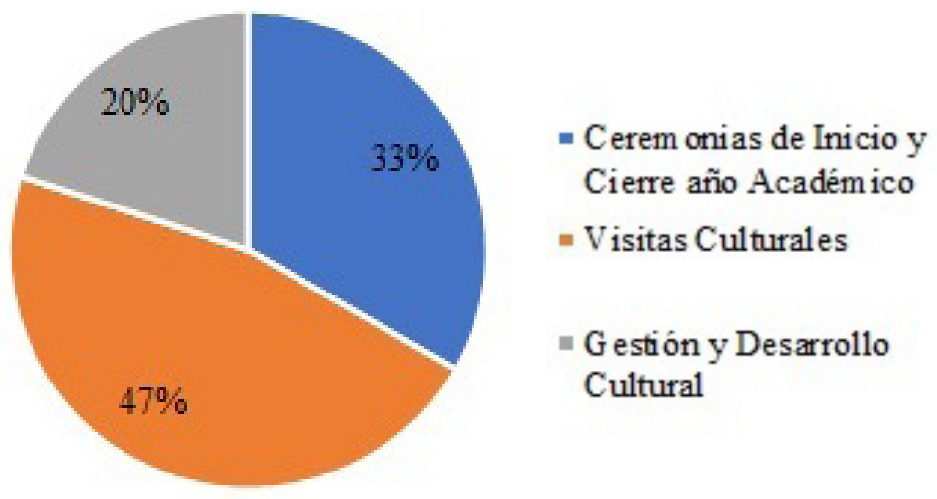

Fuente: Elaboración propia.

En el gráfico de la Fig. 6, en el ámbito Recreativo-Cultural se imparten 15 actividades distribuidas en las 11 Universidades. Un 47\% de ellas corresponde a Visitas Culturales, un 33\% a Ceremonias de Inicio y Cierre de año Académico y un 20\% refiere a acciones destinadas a vincular a la Gestión y Desarrollo Cultural. Dicho de otro modo, el foco de este conjunto de actividades está en la relación de aspectos de gestión y culturales. 


\section{Modelos de gestión}

La mayoría de los Programas de Acceso Especial se gestiona desde un modelo centrado en la reutilización de otras capacidades institucionales, además de considerar recursos profesionales y administrativos. Dentro de los equipos de trabajo participan académicos y profesionales, asumiendo roles directivos y ejecutivos. La distribución de éstos se presenta en la Fig. 7.

Fig. 7. Detalle de equipos de trabajo de los Programas.

25

20

15

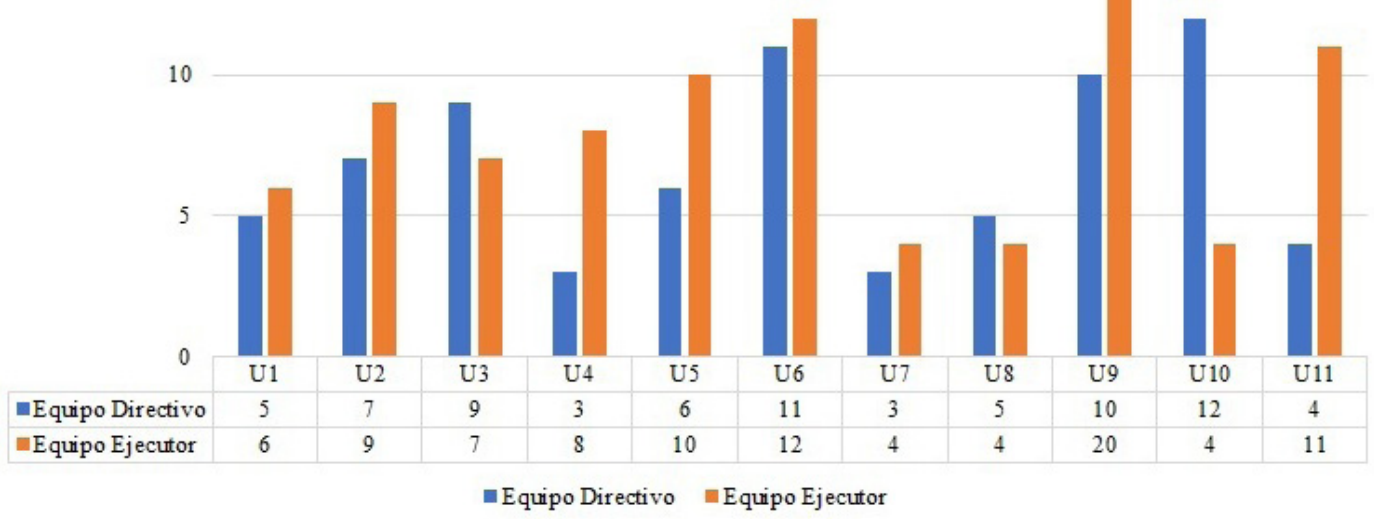

Fuente: Elaboración propia.

En términos generales, el equipo Directivo del Programa es el que se vincula con las autoridades de la Universidad y está mandatado para la toma de decisiones, el promedio de personas que lo componen es de 6. Por otra parte, el promedio de personas que integra el equipo ejecutor es 9, siendo ellos quienes coordinan el desarrollo de las actividades contempladas en los Programas.

\section{Evaluación de los Programas}

Los mecanismos implementados para monitorear los Programas se detallan a continuación (Fig. 8):

Las 11 Universidades plantean estrategias para medir la satisfacción de los estudiantes. Un 73\% utiliza encuestas de satisfacción, un 55\% Focus Group, el 18\% aplica autoevaluaciones 


\section{N. Hormazabal et al.}

y un 9\% considera de forma independiente la evaluación docente, enfoque mixto, la entrevista y estudios de tesis de pre y post grado para medir satisfacción de los Programas y recolectar información que aporte antecedentes sobre la mejora continua de éstos.

Fig. 8. Estrategias para medir la satisfacción de los Programas.

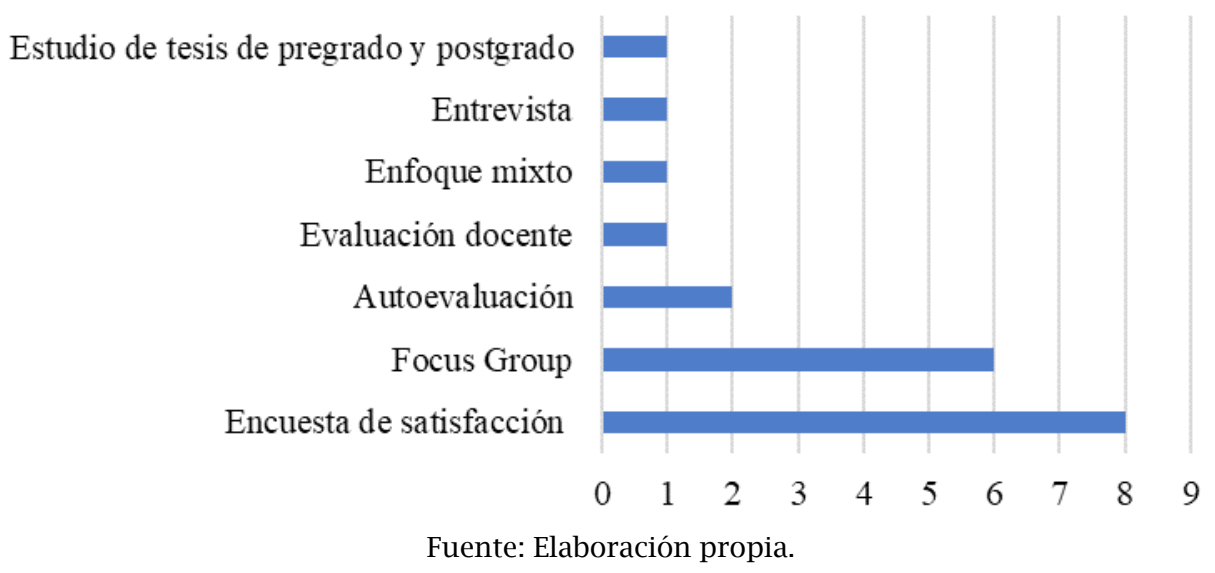

Egreso y matrícula de los estudiantes a la Universidad

Respecto a los procesos de matrícula, si bien cada casa de estudios aplica sus propias exigencias y condicionantes, se reconocen los siguientes puntos en común para permitir el acceso de los estudiantes: Aprobación del Programa, Asignación de Vacantes, Normativa institucional referida a la admisión de los estudiantes, Incorporación al calendario de Matrícula Nacional y Procesos internos de cada Universidad.

\section{Sistemas de Acompañamiento}

Los mecanismos implementados para monitorear los avances y niveles de logro de los estudiantes de enseñanza media que asistieron a los 11 Programas de Acceso Especial y que posteriormente ingresan a la universidad se especifican a continuación (Fig. 9):

En la Fig. 9 se aprecia que los Programas de acompañamiento consideran diversos ámbitos formativos. Un 82\% de ellos incluye acciones de nivelación en diversas áreas disciplinarias, un 64\% desarrolla tutorías, un 56\% contempla talleres de distinta naturaleza y un 36\% incluye acciones de acompañamiento psicosocial, al igual que técnicas de diagnóstico, caracterización y modelos predictivos. Por su parte, la nivelación en el área de matemática es utilizada por tres universidades (27\%), la inducción a la vida universitaria por dos (18\%) y la nivelación en el área de lenguaje y comunicación solamente por una de las 11 instituciones (9\%). Todos los Programas declaran que este acompañamiento opera desde el ingreso hasta el egreso, para los estudiantes que se matricularon por esta vía. 
Fig. 9. Tipos de acompañamiento de los Programas.

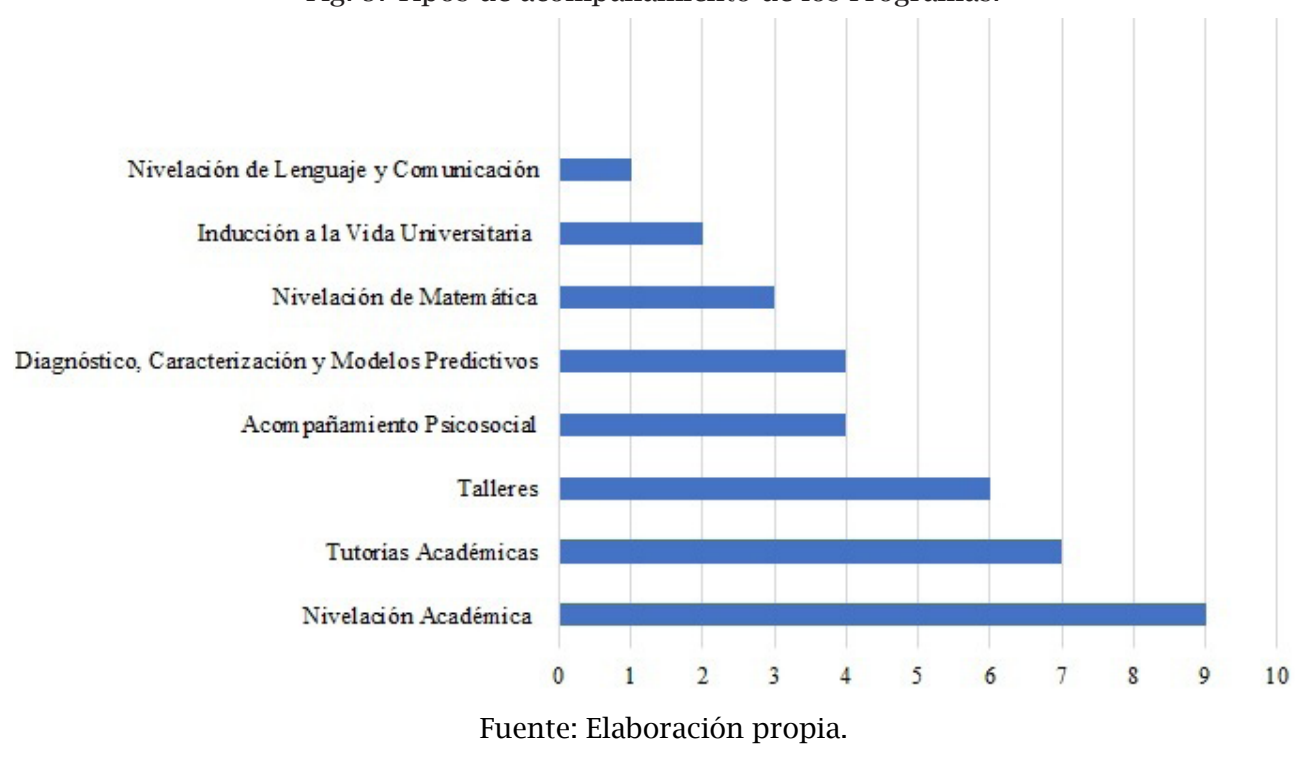

\section{Vinculación de los Programas}

La relación existente entre los programas de fortalecimiento de la equidad y vinculación con el medio, se especifican a continuación (Fig. 10):

Fig. 10. Nombre de iniciativas vinculadas con los Programas.

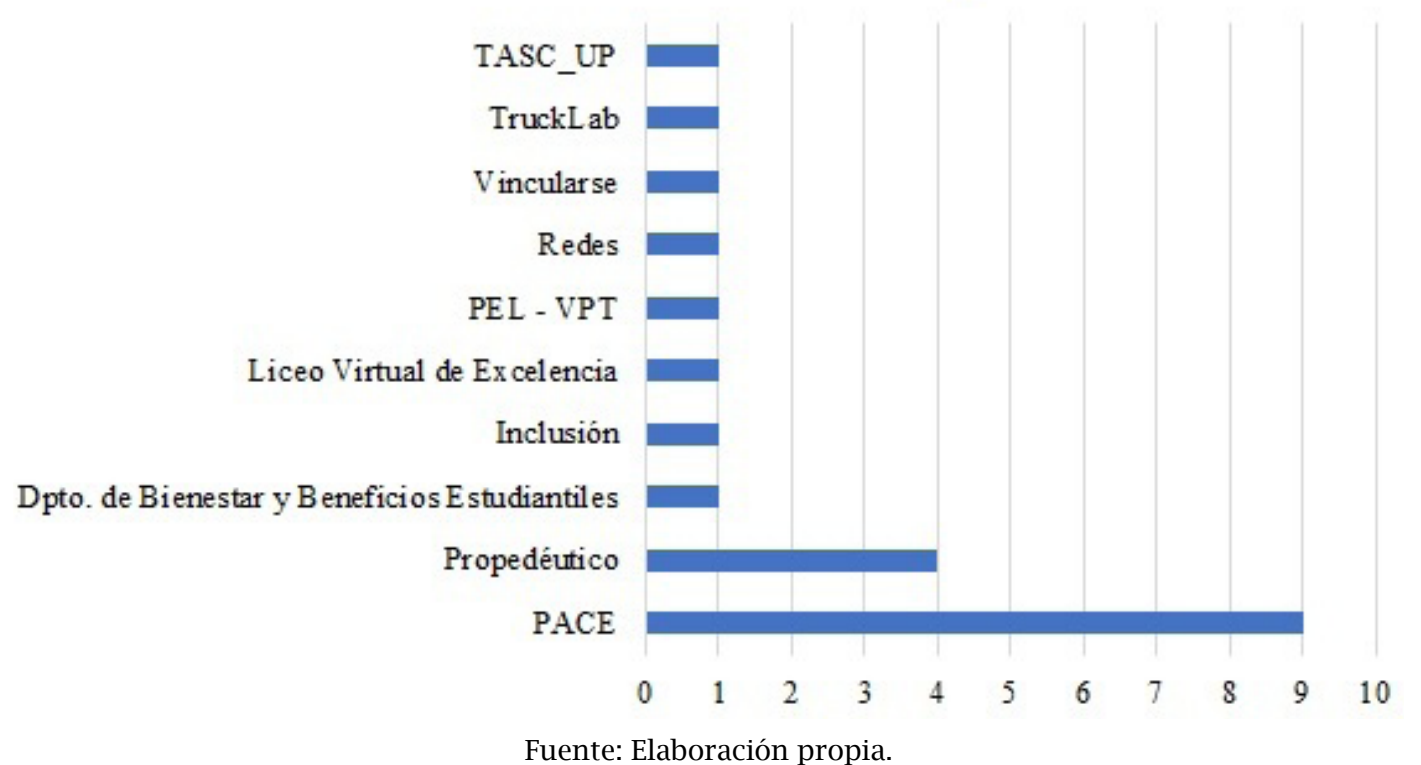

La Fig. 10 muestra claramente que el Programa de Acompañamiento y Acceso Efectivo a la Educación Superior (PACE) es el que se vincula con mayor frecuencia con los Programas de Acceso Especial a carreras de pedagogía. El 82\% de las instituciones declara un vínculo 


\section{N. Hormazabal et al.}

estrecho con dicho programa, un 36\% de las instituciones también señala relación directa con los propedéuticos que ofrece cada casa de estudios y un 9\% de las instituciones reconoce la valoración que posee con Programas específicos de cada institución de Educación Superior.

\section{Algunas contradicciones y otras buenas prácticas}

A partir de los resultados expuestos en los párrafos anteriores, los que surgen de la revisión y análisis de los Programas de Acceso, se plantea una serie de sugerencias posibles de considerar en posteriores procesos de evaluación y mejora continua de estos Programas.

La propuesta se organiza en cinco grandes ejes controversiales de los Programas: los mecanismos de difusión, su coherencia interna, la coherencia lograda con la formación inicial de profesores, la evaluación del proceso y el sistema de acompañamiento a estudiantes en la formación inicial docente.

\section{Mecanismos de difusión de los Programas}

A pesar de que existen Programas con estrategias de difusión masiva o focalizada, en todos los casos se visualiza la necesidad de aunar esfuerzos para consignar un sistema de difusión que permita a cada Programa de Acceso ser reconocido a nivel local y regional.

Complementariamente, podría ser relevante y efectivo generar una estrategia de articulación directa con los centros escolares, con la finalidad de establecer y robustecer relaciones de colaboración interinstitucional, que incluso pudieran trascender a procesos como prácticas profesionales de las carreras, formación continua, entre otros. Algunas instituciones han definido programas altamente exitosos en esta línea, desarrollados por equipos consolidados y muy conectados con los centros escolares, permitiendo generar información cruzada y acciones comunes que favorecen la formación de los estudiantes..

\section{Coherencia interna de los Programas}

En el proceso de análisis se han identificado problemáticas específicas que han movilizado el desarrollo de este tipo de Programas, de acuerdo a la realidad de cada región del país. Sin embargo, dichas dificultades descritas en los documentos, no necesariamente se alinean directamente con los objetivos o métodos utilizados en los Programas.

Algunas universidades se refieren a la necesidad de resolver la escasez de profesores en ciertas áreas como ciencias o educación parvularia (AEqualis, 2019). En esa perspectiva, se podría pensar que la Universidad probablemente estará interesada en reconocer a estudiantes con interés particular por determinadas disciplinas, así como su motivación específica por aprender a enseñar en esos ámbitos. Sin embargo, los propósitos o acciones no necesariamente están alineados en esa perspectiva. Por tal razón, se sugiere definir mecanismos que 
permitan abordar las problemáticas o desafíos planteados. Por ejemplo, en ese tipo de casos, el Programa debiese considerar aspectos específicos sobre el rendimiento académico de áreas en particular (ranking o promedio de notas), cartas de recomendación, entrevistas, simulaciones de actividades pedagógicas, relación y desarrollo de acciones con docentes destacados en ciertas áreas académicas (internados, ferias científicas, exposiciones, debates, visitas de egresados, concursos, entre otros).

Sin excluir la problemática anterior, es posible que algunas instituciones estuvieran preocupadas porque sus egresados permanecen poco en el sistema escolar (CIAE, 2018). Con ese fin, se podrían implementar mecanismos de selección que logren identificar, con mayor precisión, la motivación inicial de los estudiantes, intentando mantenerla a lo largo de la formación docente. Para esto algunos programas han procurado generar un vínculo directo con los profesores en ejercicio, con el propósito de lograr una efectiva selección de los estudiantes. En esas instancias, además de la recomendación consciente del profesor, los estudiantes podrían desarrollar entrevistas situacionales, observar actuaciones y desempeños individuales o grupales y responder instrumentos actitudinales, válidos y confiables. Un ejemplo de esto último es el instrumento FIT CHOICE (Gratacós \& López Jurado, 2016), que reconoce las motivaciones de los estudiantes al escoger las carreras de pedagogía como profesión y que está siendo aplicado por varios de los programas analizados.

Si el Programa de Acceso tiene como propósito promover la movilidad social en estudiantes y profesionales de pedagogía, se podría considerar otorgar mayor ponderación al mérito académico en contexto (Ranking). En este caso, algunos programas sugieren actividades como talleres prácticos vocacionales a cargo de profesores titulados de la institución (sesiones vocacionales y socioprofesionales), como también acciones que conducen al empoderamiento del rol transformador del profesor (ej. reconocer lo que es ser el adulto responsable frente a la infancia y adolescencia). Además, sería conveniente y muy formativo abordar las responsabilidades y beneficios de la carrera profesional docente, incluyendo actividades que promuevan el desarrollo de recursos afectivos y psicosociales.

\section{Coherencia con la formación inicial de profesores}

Se advierten algunos programas desvinculados de la realidad propia de las carreras de pedagogía. Ante esta realidad, se sugiere generar vínculos estrechos con las carreras de pedagogía de las Universidades, evitando la hiper-centralización de los Programas. La idea es vincular el quehacer y perspectiva de los Programas de estudio con la curiosidad pedagógica de los estudiantes, con el fin de fomentar su interés y motivación por las áreas disciplinares, desde la reflexión y el quehacer práctico. A modo de ejemplo, se sugiere desarrollar "aulas abiertas" en las carreras de pedagogía, vivenciar internados científicos y/o trabajos de campo, en instituciones educativas. 
Algunos programas consideran informativos vocacionales dirigidos a cuidadores/padres y orientador del Establecimiento educacional, como también talleres prácticos vocacionales a cargo de profesores titulados de la institución (vocacionales y socioprofesionales). Además, contemplan acciones destinadas a reconocer políticas públicas e institucionales referidas al seguimiento y monitoreo post egreso/titulación.

\section{Evaluación del proceso}

Todos los Programas consideran mecanismos de evaluación del proceso, entendida ésta como un método de aseguramiento de la calidad, desde un punto de vista cualitativo. Sin embargo, muchos de ellos plantean la evaluación como un proceso final, en general reducido a encuestas de satisfacción. En este sentido, podría ser relevante incluir estrategias de evaluación formativa, tal como algunos programas lo sugieren. Por ejemplo, implementar grupos focales dirigidos a estudiantes participantes de los Programas, análisis de sus producciones o entrevistas focalizadas. De igual manera algunos programas proponen incorporar las percepciones de los agentes clave de establecimientos educacionales (directivos, orientadores, profesores jefes, apoderados) y de estudiantes egresados del Programa en calidad de alumno regular de las carreras de pedagogía. En esta dinámica, se pueden distinguir los motivos o causas de abandono de los estudiantes al Programa e identificar oportunidades de mejora.

Adicionalmente, varios de los programas también sugieren considerar atención al porcentaje de asistencia, a los resultados de evaluaciones de los módulos de preparación, a la tasa de aprobación y reprobación del Programa, a la tasa de estudiantes egresados y efectivamente matriculados en carreras de pedagogía. Asimismo, proponen realizar un seguimiento longitudinal de la trayectoria académica y vocacional del estudiante (enseñanza media, pregrado y desarrollo profesional docente).

\section{Acompañamiento a estudiantes en la FID}

El área de acompañamiento a estudiantes constituye en la formación inicial docente un pilar fundamental; sin embargo, ésta cuenta con menos diversidad y riqueza de acciones en los Programas.

Por tanto, los Programas tienen que facilitar el acceso de los estudiantes a apoyos académicos específicos a cargo de pares, profesionales y académicos. Para ello, la información efectiva y oportuna resultaría determinante para implementar este tipo de acciones, como también para lograr horarios y espacios compatibles que faciliten su implementación. También sería importante capacitar a profesionales que se responsabilicen del acompañamiento y fortalecimiento de habilidades de estudiantes tutores, para que apoyen a los beneficiarios de los Programas, a lo menos en la etapa inicial de su formación. La creación de unidades, 
implementadas con actividades de aprendizaje de uso complementario, al igual que la aplicación de talleres que permitan orientar y apoyar la motivación docente, debieran ser parte de este acompañamiento.

\section{CONCLUSIONES}

Formar al profesorado involucra detectar habilidades, cualidades e intereses que podrían ser particularmente difíciles de identificar en la etapa escolar de los estudiantes. Esto resulta especialmente complejo en un contexto social que ha tensionado de forma específica la profesión docente, desde la baja valoración social de la profesión y sus malas condiciones laborales. Es por ello por lo que la detección de necesidades en los diferentes contextos geográficos y escolares es muy relevante a la hora de levantar indicaciones y sugerencias para el diseño, implementación y evaluación de los Programas de Acceso Especial a las carreras de pedagogía.

La apuesta de las políticas públicas en los últimos años ha intentado compensar, en cierta medida, dichas dificultades. Sin embargo, en lo que respecta a las Universidades Estatales, no existe una sistematización de las divergencias que oriente en cómo están aproximándose al cumplimiento de dicha política. Asimismo, las evidencias sobre la implementación de las políticas requieren ser miradas con sistematicidad y responsabilidad, aún más si los requisitos para estudiar pedagogía serán gradualmente rigurosos a partir del año 2020.

El análisis de las categorías arroja información detallada en cuanto a las acciones que las Universidades Estatales desarrollan en la implementación de sus Programas de Acceso Especial a pedagogía. Éstos consideran objetivos, forma de admisión, mecanismos de diagnóstico, actividades y módulos de preparación, modelo de gestión, evaluación de los Programas, egreso y matrícula de los estudiantes a las Universidades, sistema de acompañamiento y vinculación con otros Programas al interior de la institución. Adicionalmente, se analizan algunas controversias sobre la implementación de los programas y prácticas que podrían contribuir a abordar dichas controversias.

Los Programas de Acceso Especial a las carreras de pedagogía requieren ser implementados cuidadosamente y con pertinencia, teniendo claridad específica sobre sus propósitos y diseños, con el fin de lograr fortalecer la selección de los mejores aspirantes a profesores que el país necesita. El análisis temprano de las características de los Programas da cuenta de un planeamiento poco sistémico, algo ecléctico y con escasos referentes interinstitucionales. En cuanto al MINEDUC, este organismo ha convocado a las universidades para diseñar e implementar Programas de Acceso con la finalidad de preparar a los estudiantes respecto a "educación, talento y vocación pedagógica", como también para que se desenvuelvan efectivamente en el contexto universitario. A su vez, las instituciones aplican dispositivos de corte académico que apuntan a la nivelación de competencias, tanto disciplinares como pedagógicas. Los beneficiarios de estos Programas se preparan y 


\section{N. Hormazabal et al.}

avanzan en diversas áreas del conocimiento, dependiendo de las ofertas formativas de las Universidades Estatales.

Este artículo, que se ha limitado al análisis de los Programas de Acceso Especial de las Universidades Estatales, constituye un primer paso en la sistematización e identificación de controversias específicas detectadas en su diseño, como también de cierto nivel de incoherencia respecto a las necesidades locales. No obstante, también se descubre que se estructuran en ciertos ejes clave desde los cuales logran aportar a la formación inicial de los futuros profesores de Chile.

La caracterización de los Programas de Acceso Especial a las Pedagogías de las Universidades Estatales realizada en este estudio contribuye a la generación de una línea base respecto a su implementación en un período de tres años. Se requiere, con urgencia, sumar estudios de esta naturaleza para promover la mejora continua de este tipo de política pública, en el contexto nacional.

\section{AGRADECIMIENTOS}

Se hace mención de agradecimientos a los siguientes profesionales que también son parte del equipo de la mesa de "Programas de Acceso" de la Red Interuniversitaria Estatal de Formación de Profesores y que colaboraron en alguna de las etapas de la elaboración del manuscrito, ya sea en el apoyo de levantamiento de información teórica, sistematización de la información o contribución en la redacción del manuscrito:

- Rocío Fernández Ugalde, Psicóloga. Máster en Estudios en Políticas Educativas. Universidad de Santiago de Chile.

- Esteban Maldonado Cortez, Profesor de Historia y Ciencias Sociales. Orientador Educacional, Vocacional y Laboral. Universidad de Valparaíso.

- Karla Campaña Vilo, Profesora de Historia y Geografía. Doctora en Educación. Universidad de La Serena.

- Amalia Rojas Varela, Profesora de Historia y Geografía. Diplomado en Gestión del Patrimonio. Universidad de Tarapacá.

- María Teresa Castañeda Díaz, Profesora de Historia y Geografía. Doctora en Educación. Universidad del Bío-Bío.

\section{REFERENCIAS}

AEqualis. (2019). Proyección de números de titulados de carreras de pedagogías en Chile hacia 2023. Foro de educación superior.

Araya, J., \& Wong, T. (2013). Programa de educadores líderes con vocación pedagógica temprana de la Universidad de Santiago de Chile. En Tercera conferencia latinoamericana 
sobre el abandono en la educación superior. México DF - México.

Arias, P., \& Villaroel, T. (2019). Radiografía a las carreras de pedagogía y propuestas para maximizar el impacto dela ley de Desarrollo Profesional Docente. (p. 24) [Reporte]. Acción Educar.

Ávalos, B. (2011). Teacher professional development in teaching and teacher education over ten years. Teaching and teacher education, 27, 10-20.

Ávalos, B. (2014). La formación inicial docente en Chile: Tensiones entre políticas de apoyo y control. Estudios pedagógicos, 40, Especial, 11-28.

Banco Interamericano del Desarrollo. (2011). Estudio sobre la percepción que tienen los jóvenes de $3^{\circ}$ y $4^{\circ}$ medio sobre las carreras de educación, la experiencia laboral docente y la disposición a estudiar pedagogía. Elige Educar, 1-46.

Barber, M., \& Mourshed, M. (2008). Cómo hicieron los sistemas educativos con mayor desempeño del mundo para alcanzar sus resultados. Profesorado, Revista de Curriculum y Formación del Profesorado, 41, 301-303.

Borgerding, L. (2015). Recruitment of Early STEM Majors into possible secondary science teaching careers: the role of science education summer internships. International journal of environmental \& sciencia education, 10(2), 247-270.

Bruns, B. \& Luque, J. (2014). Profesores excelentes: cómo mejorar el aprendizaje en América Latina y el Caribe. Washington, DC.: Banco Mundial.

Cabezas, V., \& Claro, F. (2011). Valoración social del profesor en Chile: ¿cómo atraer a alumnos

talentosos a estudiar pedagogía? Temas de la agenda pública, Centro de Políticas Públicas Pontificia Universidad Católica de Chile. 42, 5.

Centro de Investigación Avanzada en Educación. (2018). Evolución de la oferta de formación docente en carreras de Pedagogía en Educación Media. CIAE.

Darling-Hammond, L., Wei, R. C., Andree, A., Richardson, N., \& Orphanos, S. (2009). State of the profession. The Learning Professional, 30(2), 42.

Darling-Hammond, L. (2000). Teacher Quality and Student Achievement: A Review of State Policy Evidence. 44.

Flores \& L. Niklasson (2014). Why do student teachers enrol for a teaching degree? A study of teacher recruitment in Portugal and Sweden. Journal of Education for Teaching. 40(4), 328-343.

Foladori, H. (2009). Hacia el análisis vocacional grupal. Revista Investigaciones en Educación,. U. de Chile, Santiago de Chile, 10, 210.

García-Huidobro, J. E. (2011). La política docente hoy y la formación de profesores. Revista Docencia, 43, 12-22.

García-López, R. (2003). Formación del profesorado en pedagogía intercultural: contenidos actitudinales. Estudios sobre educación. 4, 47-66.

Giersch, J. (2016). Academic Tracking, High-Stakes Tests, and Preparing Students for 


\section{N. Hormazabal et al.}

College: How Inequality Persists Within Schools. Educational Policy, 32(7), 907-935.

Gore, J., Barron, R. J., Holmes, K., \& Smith, M. (2016). Who says we are not attracting the best and brightest? Teacher selection and the aspirations of Australian school students. The Australian Educational Researcher, 43(5), 527-549.

Gratacós, G., \& López Jurado, M. (2016). Validación de una versión en español de la escala de los factores que influyen en la elección de los estudios de educación (FIT choice). Revista de Educación, 372, 87-110.

Hattie, J. (2009). The black box of tertiary assessment: an impending revolution. In L. H. Meyer, S. Davidson, H. Anderson, R. Fletcher, P. M. Johnston \& M. Rees (Eds.), Tertiary Assessment \& Higher Education Student Outcomes: Policy, Practice \& Research (pp. 259-275). Wellington, New Zealand: Ako Aotearoa.

Holland, J. L. (1977). Manual for the Vocational Preference Inventory. Palo Alto, California, E.U.: Consulting Psychologist Press.

Ley $\mathrm{N}^{\circ}$ 19.961. Diario Oficial de la República de Chile. Santiago, Chile, 28 de enero de 2000.

Ley N²0.129. Diario Oficial de la República de Chile. Santiago, Chile, 23 de octubre de 2006.

Ley $N^{\circ}$ 20.903. Diario Oficial de la República de Chile. Santiago, Chile, 04 de marzo de 2016.

Ling, L. M., \& Mackenzie, N. M. (2015). An australian perspective on teacher professional development in supercomplex time. Psychology, Society, \& Education, 7, 264-278.

Manuel, J., \& Carter, D. (2016). Sustaining Hope and Possibility: Early-Career English Teachers' Perspectives on Their First Years of Teaching. English in Australia, 51, 91-103.

McKenzie, P., \& Santiago, P. (2005). Teachers matter. Attracting, developing and retaining effective teachers. OECD. Francia.

Mizala, A., Hernández, T., \& Makovec, M. (2011). Determinantes de la elección y deserción en la carrera de pedagogía. Proyecto FONIDE NF511059 Santiago de Chile: Mineduc. Recuperado de: http://tinyurl. com/mizala2011.

Neugebauer, M. (2015). Who chooses teaching under different labor market conditions? Evidence from West Germany, 1980-2009. Teaching and Teacher Education, 45.

Ng, P. T., Lim, K. M., Low, E. L., \& Hui, C. (2018). Provision of early field experiences for teacher candidates in Singapore and how it can contribute to teacher resilience and retention. Teacher Development. 1-19. https://doi.org/10.1080/13664530.2018.1484388.

Núñez, I. (2007). La profesión docente en Chile: saberes e identidades en su historia. Revista Pensamiento Educativo, 41(2), 149-164.

Palazzo, J., \& Gomes, C. A. (2012). Origens sociais dos futuros educadores: a democratização desigual da educação superior. Avaliação: Revista da Avaliação da Educação Superior, 17(3), 877-898.

Quintana, J. (2014). “La decisión más importante”: estudio cualitativo sobre el proceso 
de elección vocacional en estudiantes universitarios provenientes de liceos municipales no emblemáticos de la región metropolitana. Tesis para optar al grado de Magíster en Psicología Educacional. Universidad de Chile.

Reimer, D., \& Dorf, H. (2014). Teacher recruitment in context: Differences between Danish and Finnish beginning teacher education students. Scandinavian Journal of Educational Research, 58(6), 659-677.

Rodríguez, C., \& Castillo, V. (2014). Calidad en la formación inicial docente: los déficits de las competencias pedagógicas y disciplinares en Chile. Revista Electrónica Actualidades Investigativas en Educación, 14(2), 1-25.

Rubilar, F., Rodríguez, A., \& Maldonado, A. (2017). Evaluación de la alfabetización digital y pedagógica en TIC, a partir de las opiniones de estudiantes en Formación Inicial Docente. Educação e Pesquisa, 43(1) 127-143.

Ruffinelli, A., \& Guerrero, A. (2009). Círculo de segmentación del sistema educativo chileno: destino laboral de egresados de Pedagogía en Educación Básica. Calidad en la Educación, 31, 20-44.

Ticky, L. (2016). The Future of Education for All as a Global Regime of Educational Governance. Comparative Educational Review, 61(1), 22-57.

UNESCO (2013). A teacher for every child: Projecting global teacher needs from 2015 to 2030. Montreal: UNESCO Institute for Statistics. En http://www.uis.unesco.org/Education/ Documents/fs27-2013-teachers-projections.pdf.

Vaillant, D. (2013). Formación inicial del profesorado en América Latina: dilemas centrales y perspectivas. Revista española de educación comparada, 22, 185-206.

Valenzuela, J. P., \& Sevilla, A. (2013). La movilidad de los nuevos profesores chilenos en la década del 2000: un sistema escolar viviendo en peligro (Fondecyt $\mathrm{N}^{\circ} 1120740$ ). Santiago de Chile: CONICYT.

Waissbluth, M. (2018). Educación para el siglo XXI. Fondo de Cultura Económica. 
